\title{
Altern ist Leben - Ist es auch finanzierbar?
}

\author{
Gunther Tichy*
}

The ageing of the European population gave rise to heated discussions. Most participants recommended severe reductions of public pensions, a shift towards a funded system and increased savings of the current generation to reduce the burden of the next one. The paper elaborates five counterarguments: (I) The total burden will not increase much, as the additional pensioners are partly compensated by a reduced number of unemployed. (2) Even with I percent growth per capita GDP will be some 60 percent higher in 2050, which should suffice to increase the living standard of the old and the young. (3) The real burden cannot be shifted, and a shift of the financial burden can prove counterproductive. (4) The pay-as-you-go system should not be abandoned, as it covers wider and more severe risks than a funded one. (5) Surveys show clearly that the population appreciates a public pension scheme and prefers higher contributions to reduced pensions.

JEL classifications: $H_{55}, J_{I I}, J_{I 4}, J_{26}$

Dass die Bevölkerung in allen Industrieländern durch das Zusammentreffen sinkender Fertilität mit höherer Lebenserwartung schrumpft, der Anteil der Älteren somit zunimmt, sodass eine geringere Zahl "Junger" eine größere Zahl "Alter« erhalten muss, und dass dieser Trend durch das Altern der Babyboom-Generation massiv verstärkt wird, ist weithin in das Bewusstsein einer breiten Öffentlichkeit gedrungen. Die Folgen dieser Entwicklung werden jedoch vielfach unrichtig eingeschätzt: Abgesehen davon, dass demografische und Finanzierungsaspekte überschätzt und überbetont werden, ergibt sich das aus der bewussten

* Institut für Technikfolgenabschätzung, Österreichische Akademie der Wissenschaften. Der Autor dankt Alois Guger und Christine Mayrhuber für wertvolle Kommentare zu einer früheren Fassung sowie zwei anonymen Gutachter|inne|n für Verbesserungsvorschläge.

Korrespondenzadresse:

Prof. Dr. Gunther Tichy, Institut für Technikfolgen-Abschätzung, Österreichische Akademie der Wissenschaften, Strohgasse 45, A-IO30 Wien, Österreich, E-Mail: gtichy@oeaw.ac.at

Ersteinreichung des Artikels am 16.05.2005, endgültig akzeptiert am 05.08.2005

(C) InTERVENTION. Zeitschrift für Ökonomie, Jg. 2 (2005), H. 2, S. 107-130 
Umlenkung der Diskussion auf vielfach ganz andere Ziele: Verantwortungslose Publizist|inn|en versuchen aus dem Schlagwort, dass künftig jeder "Junge« doppelt so viele »Alte« erhalten müsste, einen Generationenkonflikt um die Verteilung zu schüren (vgl. Tichy 2004b), neokonservative Politiker|innen sehen eine Chance, traditionelle Staatsaufgaben billig loszuwerden, neokonservative Ökonom|inn|en glauben, Möglichkeiten der Effizienzsteigerung durch Reduzierung der Umverteilung zu erkennen, und die Finanzwirtschaft versucht von dem enormen Geschäftspotential einmal abgesehen - die Chance wahrzunehmen, ihre Macht erheblich zu stärken, indem sie den Kapitalmarkt als zentrales Steuerungselement

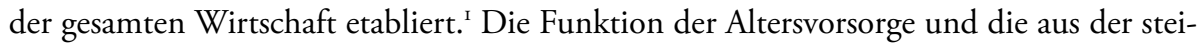
genden demografischen Belastungsquote resultierenden Probleme bleiben dabei weitgehend auf der Strecke.

Es kann und soll nicht Aufgabe dieser Arbeit sein, die unzähligen Gutachten und Detailvorschläge einer Reform der Altersvorsorge zu diskutieren; sie beschränkt sich bewusst auf vier grundlegende Fragen: Führt die steigende demografische Belastung zwangsläufig zu einer entsprechenden ökonomischen Belastung? Ist eine lebensstandardsichernde Altersversorgung in den nächsten fünf Dekaden wirklich unfinanzierbar? Kann eine Umstellung von der gegenwärtigen Umlagefinanzierung auf zumindest partielle Kapitaldeckung die Probleme lösen? Und: Wie sehen die Betroffenen die Zukunft ihrer Altersversorgung? Die Antworten auf diese Frage zeigen einige Aspekte auf, die in der wissenschaftlichen wie in der politischen Diskussion vernachlässigt werden: Erstens, dass im Gegensatz zur demografischen Unterstellung nicht "Junge«"Alte« erhalten, sondern Arbeitende NichtArbeitende, also auch Arbeitslose und Frühpensionist|inn|en; die "volkswirtschaftliche Belastungsquote« ist daher derzeit viel höher als die demografische, und sie wird weniger stark steigen, weil das Schrumpfen der Bevölkerung im erwerbsfähigen Alter die Zahl der Arbeitslosen und Frühpensionist|inn|en reduzieren wird. Zweitens wird die Finanzierung der künftigen Pensionen darüber hinaus dadurch erleichtert, dass auch bei langsamerem Wachstum der Wirtschaft Produktion und Einkommen und damit der Wohlstand in einem Maße zunehmen werden, dass sowohl Junge wie Alte besser leben können als heute. Reformen sind zwar unabdingbar, doch netto können Alle, "Junge« wie »Alte«, besser gestellt sein. Drittens zeigt sich, dass das Umlageverfahren die Altersversorgung stärker absichert als ihre Alternativen: Allein diese Finanzierungsform kann die wirklich großen Risiken (Krieg, galoppierende Inflation) absichern, allein das Umlageverfahren setzt die Versicherten nicht dem Kapitalmarkt-(Zins-, Aktienkurs-)risiko aus und verhindert die Kapitalakkumulation bei großen institutionellen Anlegern, die in den USA zur Steuerung der gesamten Wirtschaft über den Kapitalmarkt (shareholder value) zu Lasten der übrigen stakeholder und zu short

I Während in Europa Finanzierungsängste möglicherweise wirklich die treibenden Elemente der Pensionsdebatte sind, stehen in den USA offensichtlich primär Geschäftsinteressen des Finanzsektors hinter dem Druck auf volle Privatisierung und Kapitaldeckung der Altersvorsorge. In der über hundertseitigen Unterlage "Saving Social Security « wird Präsident Bush bei seiner Kampagne zu »einfacher Sprache« und zur anschaulichen Welt »kleiner Zahlen« sowie zur Vermeidung des Begriffs »Privatisierung" geraten (vgl. Fischermann 2005a, b, c). Siehe dazu auch Fußnote 23. 
terminism geführt hat. Viertens lassen Umfragen deutlich erkennen, dass die europäischen Bürger|innen - entgegen pausenloser Beteuerung durch Politiker|innen und Massenmedien Umverteilung generell wie auch Umverteilung zwischen "Jung " und "Alt" nicht bloß akzeptieren, sondern sogar wünschen; sie sind bereit für soziale Sicherheit erforderlichenfalls auch mehr zu bezahlen, sie erwarten Reformen und sind weitgehend bereit, deren Konsequenzen zu tragen.

\section{Wider den Mythos der Unfinanzierbarkeit}

Die Standardargumentation der üblichen Pensionsdebatte - in Wissenschaft wie Politik ${ }^{2}$ geht von der demografischen Altersbelastungsquote aus, der Relation der Bevölkerung im erwerbstätigen Alter zu denen jenseits des erwerbstätigen Alters: Erhalten heute in Europa vier bis fünf 20- bis 65-Jährige einen über 65-Jährigen, werden es 2050 nur noch zwei bis drei sein. Das ist erschreckend - und scheinbar plausibel. Das Argument übersieht jedoch, bewusst oder unbewusst, dreierlei: Erstens, dass die jeweils arbeitende Generation nicht bloß die "Alten", sondern auch die Kinder erhalten muss; da deren Zahl jedoch deutlich abnimmt und noch weiter abnehmen wird, verringert das die Belastung der Arbeitenden. Zweitens wird ignoriert, dass infolge der gedämpften Wirtschaftsentwicklung in Europa derzeit rund ein Zehntel des Arbeitskräftepotentials arbeitslos ist und viele über 50-Jährige aus dem Erwerbsleben ausgeschieden sind; die angesichts des künftigen Arbeitskräftemangels zu erwartende Beschäftigung dieser Personen wird die Erwerbsquote erhöhen und die gesamtwirtschaftliche Belastungsquote weniger stark steigen lassen, als es der demografischen Komponente entspräche. Drittens vernachlässigt die laufende Argumentation, dass in den nächsten 20 bis 50 Jahren nicht bloß die Zahl der "Alten" zunehmen wird, sondern - mit an Sicherheit grenzender Wahrscheinlichkeit - werden auch die Einkommen zunehmen. Diese drei Einwände gegen die übliche Argumentation sollen im Folgenden mit österreichischen Daten belegt werden. ${ }^{3}$

\section{I.I Die demografische Belastung unterschätzt die tatsächliche}

Die demografische Altersbelastungsquote unterschätzt die derzeitige Belastung erheblich und überschätzt die Dramatik deren Erhöhung. Erstens wird sich - wie Abbildung I auf der folgenden Seite zeigt - zwar der Anteil der über 6o-Jährigen in Österreich gemäß der mittleren Variante der Bevölkerungsprognose zwischen 2000 und 2050 fast (von 15,5 Prozent auf 28,5 Prozent) und der der über 65-Jährigen mehr als verdoppeln (von I0,5 Prozent auf 22 Prozent), der Anteil der Bevölkerung im erwerbsfähigen Alter wird jedoch keineswegs tung durch Alter sowie durch geringe Alters-Erwerbsquoten ist hier etwas höher, die durch Arbeitslosigkeit etwas geringer als in den meisten anderen europäischen Staaten. 
Abbildung Ia und Ib: Demografische Belastung nach Pensionsantrittsalter (6o bzw. 65 Jahre, Skala zwecks Verdeutlichung unten abgeschnitten)
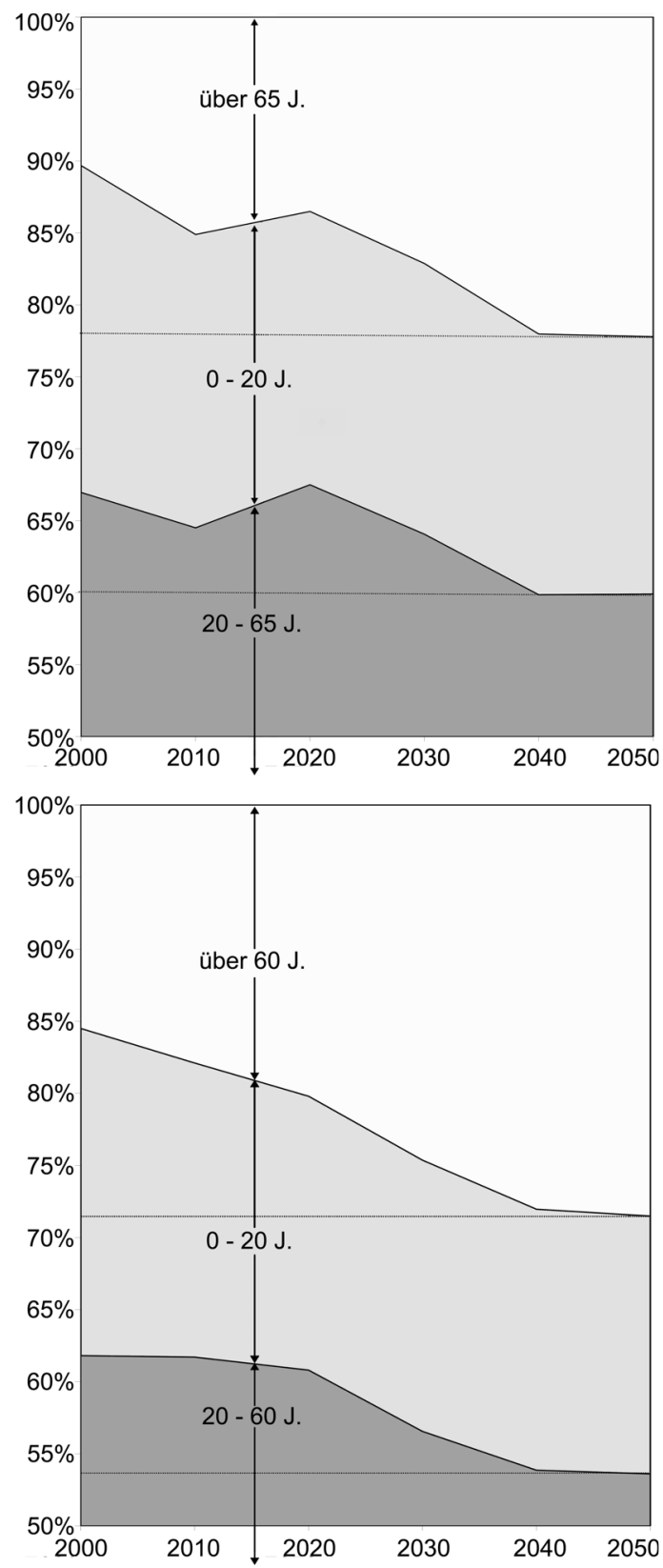

Quelle: Statistik Austria 2004, Tab. 2.09 
Abbildung 2: Demografische Belastung aus längerfristiger Perspektive

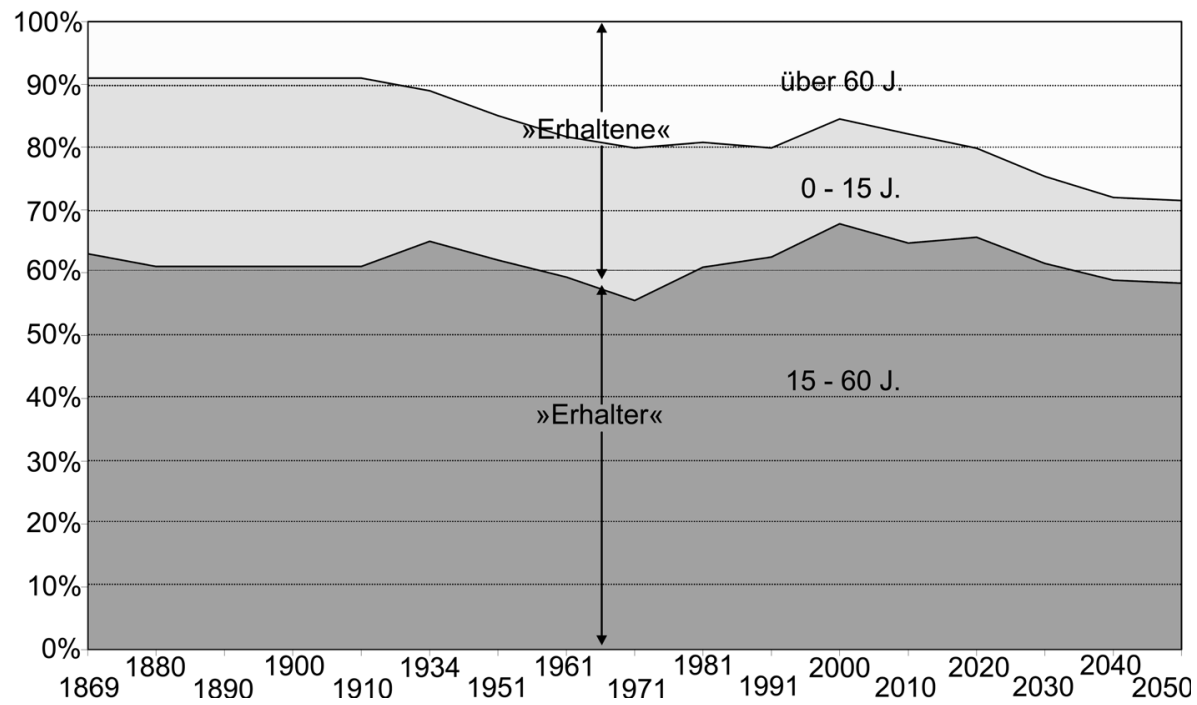

Quelle: Statistik Austria 2004, Tab. 2.08, 2.09

proportional dazu abnehmen, ${ }^{4}$ weil der Anteil der unter 20-Jährigen von 22,5 Prozent auf I8 Prozent schrumpft.

Abbildung 2 lässt darüber hinaus erkennen, dass die künftige Entwicklung im historischen Vergleich keineswegs besonders ungewöhnlich ist: In der Vergangenheit lag die demografische Gesamtbelastungsquote zumeist um die 6o Prozent; atypisch ist somit nicht das künftige Niveau der Belastungsquote, atypisch sind eher die günstigen Werte in den I990er Jahren. Allerdings darf nicht verschwiegen werden, dass für Kinder primär im Rahmen der Familie (privater Konsum), für Pensionist|inn|en hingegen überwiegend kollektiv (Zwangsabgaben) vorgesorgt wird, und dass Kinder eher »billiger « sind als Pensionist|inn|en. ${ }^{5}$ Für die reale Belastung (der jeweiligen Produktion durch den Konsum der »Unproduktiven«) spielt das allerdings keine Rolle.

Zweitens führt die isoliert demografische Argumentation insofern in die Irre, als die Belastung nicht aus dem Kriterium "Alter«, sondern aus dem Kriterium "Nicht-Arbeiten« entsteht. Infolge der ungünstigen Arbeitsmarktlage sind derzeit eine Viertel Million Men-

4 Von 62 auf 53,5 Prozent bei einem Pensionsantrittsalter von 60 Jahren, von 67 Prozent auf 60 Prozent bei einem Pensionsalter von 65 Jahren.

5 Der Einwand darf allerdings nicht überschätzt werden: Die volkswirtschaftliche Belastung muss die Ausbildungskosten berücksichtigen - von den 25- bis 30-Jährigen hat mehr als die Hälfte Fachschul- oder höhere Ausbildung, die zum erheblichen Teil von der öffentlichen Hand getragen wird. Auch sind »Alte« insofern »billiger« als Erwerbstätige, als die Pensionen i.d.R. unter den Aktiveinkommen liegen. 
Tabelle I: Demografische und volkswirtschaftliche Belastung

\begin{tabular}{|c|c|c|}
\hline & 2000 & 2050 \\
\hline (0) Gesamtbevölkerung & 8.110 & 8.214 \\
\hline \multicolumn{3}{|l|}{ Demografische Alterbelastung } \\
\hline (1) 20- bis 65-Jährige & 5.010 & 4.402 \\
\hline (2) über 65-Jährige & 1.256 & 2.339 \\
\hline Demografische Altersbelastungsquote (2)/(1) & $25 \%$ & $53 \%$ \\
\hline \multicolumn{3}{|l|}{ Demografische Gesamtbelastung } \\
\hline (1) 20- bis 65-Jährige & 5.010 & 4.402 \\
\hline (3) 0 - bis 20 - und über 65 -Jährige & 3.100 & 3.812 \\
\hline Demografische Gesamtbelastungsquote (3)/(1) & $62 \%$ & $87 \%$ \\
\hline \multicolumn{3}{|l|}{ Arbeitsmarkt-Belastung } \\
\hline (4) Arbeitslose unter 50 Jahre & 184 & 64 \\
\hline (5) "Frühpensionisten«* & 788 & 468 \\
\hline Arbeitsmarkt-Belastungsquote $(4+5) /(1)$ & $19 \%$ & $12 \%$ \\
\hline \multicolumn{3}{|l|}{ Volkswirtschaftliche Gesamtbelastung } \\
\hline (6) "Erhalter" (1) - (4+5) & 4.038 & 3.870 \\
\hline (7) "zu Erhaltende" (0) - (6) & 4.072 & 4.344 \\
\hline Volkswirtschaftliche Gesamtbelastungsquote (7)/(6) & $101 \%$ & $112 \%$ \\
\hline
\end{tabular}

* Nicht-Erwerbstätige 50- bis 65-Jährige

Quelle: Statistik Austria (2003), Tab. 2.098, Wifo-Datenbank (eigene Berechnungen)

schen arbeitslos, und die Erwerbsquote der über 50-Jährigen liegt mit bloß 48 Prozent weit unter der der 35- bis 45-Jährigen (89 Prozent); rund 50.000 von ihnen sind arbeitslos, an die 400.000 in Frühpension. Unter der Annahme, dass die Arbeitslosenquote der unter 50-Jährigen zu zwei Drittel auf die schlechte Arbeitsmarktlage zurückgeht und die Erwerbsquote der 50- bis 65-Jährigen bei günstigerer Arbeitsmarktlage 70 Prozent erreichen könnte (was etwa den gegenwärtigen Quoten in Skandinavien entspricht), ergibt das rund eine Million Menschen, die in Österreich derzeit über die demografisch bedingte Quote hinaus "erhalten " werden müssen; zu der demografischen Gesamtbelastungsquote von 62 Prozent müsste daher eine "Arbeitsmarktbelastungsquote« von I9 Prozent hinzugezählt werden (Tabelle I), was 82 Prozent ergeben würde. Diese Kennziffer macht jedoch wenig Sinn, weil sie die "Erhaltenen « auf die Bevölkerung im erwerbsfähigen Alter bezieht (die bloß zum Teil beschäftigt ist); bezogen auf das Arbeitspotential (»Erhalter|innen«) gemäß obiger Schätzung ${ }^{6}$

6 20- bis 65-Jährige minus zwei Drittel der Arbeitslosen unter 50 minus Frühpensionist|inn|en (70 Prozent Erwerbsquote der 50- bis 65-Jährigen). 
ergibt sich schon für das Jahr 2000 eine volkswirtschaftliche Gesamtbelastungsquote von IOI Prozent, das Vierfache der demografischen Altersbelastungsquote.

Die Abschätzung der künftigen Entwicklung muss zwangsläufig von der Bevölkerungsprognose und Annahmen über Erwerbs- und Arbeitslosenquoten ausgehen. Der für diese Überlegungen relevante Teil der Bevölkerungsprognose ist relativ unproblematisch, da der größte Teil der Bevölkerung, die 2050 im erwerbsfähigen Alter sein wird, sowie alle dann "Alten« schon jetzt existieren: Gemäß der mittleren Variante der Prognose wird die Bevölkerung im erwerbsfähigen Alter 2050 um rund eine halbe Million kleiner sein, und es kann daher angenommen werden, dass der Arbeitsmarkt eher angespannt sein wird. Unter den obigen Annahmen über das Arbeitskräftepotential bei guter Arbeitsmarktlage wird die Arbeitslosigkeit um rund I20.000 niedriger und die Erwerbstätigkeit der 50- bis 65-Jährigen um 320.000 höher sein (Erwerbsquote 70 Prozent); rund 440.000 Menschen werden somit von den »Erhaltenen« zu den »Erhalter|inne|n " wechseln, was sich auf die volkswirtschaftliche Gesamtbelastungsquote doppelt auswirkt: Sie steigt demgemäss bloß von IOI Prozent auf II2 Prozent, wesentlich schwächer, als die isoliert demografische Alternsdiskussion erwarten ließe.

\section{I.2 Wachstum vergrößert die Verteilungsmasse}

Die gegenwärtige Pensionsdebatte vernachlässigt bei ihrer Bewertung der künftigen Belastung neben der (partiell) kompensierenden Abnahme der Jugendlichen, Arbeitslosen und Frühpensionist|inn|en drittens die Entwicklung der Einkommen und der Produktivität. ${ }^{7}$ Zwar wird die geringere Zahl der Arbeitenden Gesamtproduktion und -einkommen tendenziell langsamer wachsen lassen, doch wird das in seiner Wirkung auf die Pro-Kopf-Einkommen durch die Beschäftigung von bisher offen oder versteckt Arbeitslosen und die steigende Arbeitsproduktivität überkompensiert. Selbst in der Periode 1993-2002, bei keineswegs überbordender Konjunktur, stieg die gesamtwirtschaftliche Arbeitsproduktivität (BIP je Erwerbstätigem/Erwerbstätiger) um I,75 Prozent pro Jahr, und das reale Bruttoinlandsprodukt (BIP) wuchs trotz erheblicher Unterbeschäftigung um zwei Prozent p.a. In Zukunft wird die Arbeitsproduktivität eher rascher zunehmen: Infolge des künftigen Arbeitskräftemangels, der zu verstärkter Rationalisierung zwingt, aber auch, weil die in den Arbeitsmarkt Eintretenden besser ausgebildet sind als die Ausscheidenden und ihnen ein größerer Kapitalbestand (in Form von Maschinen wie Infrastruktur) zur Verfügung steht.

7 Das ist keineswegs ein neuer Gedanke: Bütler and Kirchsteiger haben 1999 auf dieses Problem hingewiesen und in einem theoretischen Modell die Frage beantwortet, wie viel technischer Fortschritt (und damit letztlich auch Wirtschaftswachstum) zur Finanzierung der demografischen Belastung erforderlich ist (vgl. Bütler/Kirchsteiger 1999). Der Autor selbst hat die österreichische Debatte schon 1988, 1999 und 2004 - erfolglos - in diese Richtung zu lenken versucht (vgl. Tichy 1988, 1999 und 2004b). 
Geht man von einem I,7-prozentigen Produktivitätswachstum und - infolge des zu erwartenden Arbeitskräftemangels - von den in Abschnitt I.I beschriebenen Annahmen über die Zahl der Erwerbstätigen aus, wird das reale BIP pro Kopf 2050 mit 57.500 € um I25 Prozent über dem von 2000 liegen (siehe Tabelle 2); jede $r$ Bürger|in, ob alt oder jung, kann daher ein um 25 Prozent höheres Einkommen haben. ${ }^{8}$ Selbst wenn man - pessimistisch - von einer Verlangsamung des Produktivitätswachstums auf bloß ein Prozent ausgeht, werden die Pro-Kopf-Einkommen 2050 noch immer um mehr als die Hälfte höher sein als heute. Die Bevölkerung wird unter diesen Annahmen daher mit hoher Wahrscheinlichkeit nicht bloß vollbeschäftigt, sondern auch wohlhabender sein. Wie der Einkommenszuwachs zwischen den Bevölkerungsgruppen und Einkommenskategorien allerdings aufgeteilt wird, bleibt auszuhandeln; dabei können die Einen oder die Anderen leer ausgehen. ${ }^{9}$ Soll die Einkommensrelation von Beschäftigten zu Pensionist|inn|en ceteris paribus gleich bleiben, müssen die Beitragssätze zur Pensionsversicherung natürlich erhöht werden. Allerdings können auch dann beide Gruppen besser leben als heute: da das BIP erheblich höher sein wird, bleibt auch bei höherer Belastung netto mehr übrig. Es gibt keinen Grund für die Befürchtung, dass der Lebensstandard der "Jungen" oder der "Alten" sinken müsse; ganz im Gegenteil.

Aus der Tatsache, dass wachstumsbedingt »Alte« wie »Junge« auch bei steigender Belastungsquote besser leben können, darf nicht geschlossen werden, dass man sich Reformen ersparen könnte. So gehen schon die obigen Schätzungen von einem Pensionsantrittsalter von 65 Jahren aus; ${ }^{\text {IO }}$ weiters impliziert ein höheres Netto-Einkommen für alle Gruppen höhere Beitragssätze und/oder eine Ausweitung der Beitragsgrundlage auch auf NichtArbeitseinkommen, bzw. - wie in Skandinavien - höhere Verbrauchssteuern. Höhere Belastung wird in der politischen Diskussion zumeist als äußerst problematisch hingestellt, doch zeigen Umfragen mit großer Deutlichkeit, dass die Bevölkerung den Wert der Sozialversicherung so hoch einschätzt, dass sie Beitragserhöhungen durchaus - wenn auch ungern akzeptiert (siehe Abschnitt 4).

\section{Hilft "Ansparen « auf die kommende Belastung?}

Eine Erhöhung der Beitragsgrundlagen oder -sätze bedeutet allerdings, dass die Belastung aus der geänderten Altersverteilung von den jeweils Arbeitenden getragen werden muss, und das wird in der öffentlichen Debatte vielfach als ungerechte Belastung der künftigen

8 Auf Grund von ökonometrischen Schätzungen kommt der Internationale Währungsfonds (2004) zu durchaus ähnlichen Ergebnissen.

9 Die "Unfinanzierbarkeit« der Pensionen ist kein Naturgesetz; es geht um die Aufteilung des künftig höheren Einkommens zwischen Firmen (Kapitalbesitzer|inne|n), Beschäftigten und Pensionist|inn|en, bzw. zwischen Reich und Arm.

IO Das ist deswegen plausibel, weil die stärkere Anspannung des Arbeitsmarkts die Frühpensionierungen stark reduzieren wird und bis 2050 auch das Pensionsantrittsalter der Frauen angeglichen sein wird. 
Tabelle 2: Entwicklung des realen Brutto-Nationalprodukts (BIP)

\begin{tabular}{l|l}
\hline $\begin{array}{c}\text { Produktivitätsanstieg 1,7\% } \\
\text { pro Jahr }\end{array}$ & \\
\hline BIP & 206,7 Mrd. $€$ \\
2000 & $472,6 \mathrm{Mrd} . €$ \\
\hline 2050 & $+129 \%$. \\
\hline Veränderung & \\
\hline Erwerbstätige & 4.457 .000 \\
\hline 2000 & 4387.000 \\
\hline 2050 & $-1 \frac{1}{2} \%$ \\
\hline Veränderung & \\
\hline BIP je Erwerbstätigen & \\
(Erwerbstätigenproduktivität) & $46.376 €$ \\
\hline 2000 & $107.731 €$ \\
\hline 2050 & $+132 \%$ \\
\hline Veränderung & \\
\hline Bevölkerung & 8.110 .000 \\
\hline 2000 & 8.214 .000 \\
\hline 2050 & $+11 \frac{1}{4} \%$ \\
\hline Veränderung & $25.519 €$ \\
\hline Pro-Kopf-BIP & $57.538 €$ \\
\hline 2000 & $+125 \%$ \\
\hline 2050 & \\
\hline Veränderung & \\
\hline & \\
\hline
\end{tabular}

\begin{tabular}{l|l}
\hline $\begin{array}{c}\text { Produktivitätsanstieg 1,0\% } \\
\text { pro Jahr }\end{array}$ & \\
\hline BIP & 206,7 Mrd. $€$ \\
2000 & 334,6 Mrd. $€$ \\
\hline 2050 & $+62 \%$ \\
\hline Veränderung & \\
\hline Erwerbstätige & 4.457 .000 \\
\hline 2000 & 4387.000 \\
\hline 2050 & $-1 \frac{1}{2} \%$ \\
\hline Veränderung & \\
\hline BIP je Erwerbstätigen \\
(Erwerbstätigenproduktivität) & \\
\hline 2000 & $46.376 €$ \\
\hline 2050 & $76.271 €$ \\
\hline Veränderung & $+64 \%$ \\
\hline Bevölkerung & 8.110 .000 \\
\hline 2000 & 8.214 .000 \\
\hline 2050 & $+11 \% \%$ \\
\hline Veränderung & $25.519 €$ \\
\hline Pro-Kopf-BIP & $40.736 €$ \\
\hline 2000 & $+60 \%$ \\
\hline 2050 & \\
\hline Veränderung & \\
\hline & \\
\hline &
\end{tabular}

Annahmen über Erwerbstätige siehe Tabelle I

Quelle: Eigene Zusammenstellung

Generation betrachtet. Daraus resultiert die Empfehlung, diese durch »Ansparen« auf die künftigen Pensionen zu entlasten: indem das Pensionssystem selbst Rücklagen bildet, oder indem die Versicherten im Zuge eines teilweisen Übergangs zu einem Kapitalstockverfahren selbst auf ihre Pension ansparen. Diese wohlmeinende Argumentation beruht zumindest teilweise auf einer Überbetonung finanzieller Überlegungen (vgl. Barr 20oI) und auf einer fälschlichen Übertragung einzelwirtschaftlicher Überlegungen auf die Gesamtwirtschaft: Realwirtschaftlich kann in jeder Periode bloß das verbraucht werden, was in derselben Periode produziert wird (vom Aufbrauchen eventueller Lager einmal abgesehen, was kurzfristig kostspielig und langfristig unmöglich ist). Dem gemäß konsumieren die Pensionist|inn|en zwangsläufig die Güter, die die Aktiven im jeweiligen Zeitraum produzieren, ob sie Sparguthaben aus ihrer Aktivzeit auflösen oder nicht; das impliziert natürlich, 
dass den Aktiven nur ein Teil ihrer eigenen Produktion bleibt - gütermäßig tragen in jedem Fall unvermeidlicherweise sie die Belastung. Die Pensionist|inn|en können zwar Finanzkapital aus ihrer Aktivzeit in die Pension übertragen, kaum jedoch Güter und noch weniger Dienstleistungen. ${ }^{\text {II }}$

Als Ausweg wird zumeist eine indirekte (reale) Übertragung gesehen: die Investition der Ersparnisse in Produktionsanlagen. Dadurch würden die künftigen Pensionist|inn|en selbst die Anlagen finanzieren, die ihre künftigen Konsumbedürfnisse befriedigen. Abgesehen davon, dass auf diese Weise Zeitspannen von 30 bis 50 Jahren überbrückt werden müssten, sodass bei dynamischem Strukturwandel die Gefahr von Fehlinvestitionen groß ist, ${ }^{\mathrm{I} 2}$ geht diese Hypothese von der Annahme generellen Kapitalmangels aus, m.a.W. davon, dass Investitionen derzeit aus Mangel an Finanzierung unterbleiben. Das mag in Perioden raschen Wachstums und hoher Beschäftigung realistisch sein, doch das ist nicht die gegenwärtige Situation; derzeit unterbleiben Investitionen nicht aus Mangel an Finanzierung, sondern aus Mangel an Auslastung, und in einer schrumpfenden und alternden Gesellschaft wird der Bedarf an Investitionen eher weiter sinken. Zusätzliches Sparen, d.h. Einschränkung des Konsums - und dadurch bedingt steigende Unterauslastung - würde die Probleme verschärfen, nicht lindern.

Ansparen auf die kommende demografische Belastung ist daher nicht bloß gütermäßig unmöglich, sondern als Folge einer unzulässigen Übertragung einzelwirtschaftlichen Denkens auf die Gesamtheit ${ }^{13}$ auch kontraproduktiv. Denn wenn der Einzelne spart, kann er im Alter davon zehren; wenn aber eine ganze Generation mehr spart, um im Alter davon zu leben, machen sich die Einzelnen den Erfolg wechselseitig streitig: Wenn alle zugleich sparen, sinkt der Zinssatz, und zwar aus zwei Gründen. Erstens, weil das Angebot an Ersparnissen steigt, und zweitens, weil die Nachfrage nach Ersparnissen sinkt - denn steigendes Sparen heißt zwangsläufig weniger Konsum, und weniger Konsum heißt weniger Bedarf an Investitionen und damit weniger Nachfrage an Ersparnissen zur Investitionsfinanzierung. Ansparen einer ganzen Generation ist daher gesamtwirtschaftlich problematisch und für den Einzelnen »unrentabel«, weil es nur zu schlechten Anlagebedingungen möglich ist (und im Extremfall sogar zu Arbeitslosigkeit führt). Und diese Falle schnappt ein zweites Mal zu, wenn die Generation der Sparer|innen im Alter von ihren Ersparnissen leben will: Dann muss sie ihre Obligationen oder Aktien verkaufen, was den Kurs drückt, wenn es alle zugleich versuchen; die Pensionist|inn|en können ihre Ersparnisse nur mit Verlust rea-

II Selbst wenn sie in ihrer Aktivzeit ausreichende Konservenbestände anlegen würden, änderte das kaum etwas; denn in viel höherem Ausmaß richtet sich die Nachfrage der Pensionist|inn|en auf nicht lagerbare Komponenten, insbesondere Dienstleistungen (Ärzte/Ärztinnen, Pflege, Unterhaltung, Reisen, etc.).

I2 Es müsste sich ja um langfristige Investitionen handeln, die die Zeitspanne von einem Viertelbis einem halben Jahrhundert überbrücken. Zusätzlich steigt die Gefahr von Fehlinvestitionen dadurch, dass das Ansparen den Zinssatz drückt und dadurch Investitionen induziert werden, die bei dem in der Entsparperiode steigendem Zinsniveau nicht mehr rentabel sind.

I3 Diese so genannte fallacy of composition wird zumeist mit dem Kinobeispiel illustriert: Wenn im Kino eine|r aufsteht, sieht er/sie besser, wenn alle aufstehen, erhöhen sie bloß ihre Unbequemlichkeit. 
lisieren (»asset melt-down hypothesis«). ${ }^{14}$ Damit führt der Versuch, gesamtwirtschaftlich auf die Finanzierung des Alters anzusparen, zu einem doppelten Vermögensnachteil: Nicht nur die Anlage kann bloß zu schlechten Bedingungen (hoher Kurs, niedriger Zinssatz) erfolgen, sondern auch die Auflösung (niedriger Kurs, hoher Zinssatz).

Gegen beide Argumente, die Unmöglichkeit des gütermäßigen Transfers wie die induzierte Verschlechterung der Anlagebedingungen, wird üblicherweise eingewendet, dass sie bloß in einer geschlossenen Wirtschaft gälten (vgl. Börsch-Supan et al. 2003); in einer offenen Wirtschaft könnten sowohl Güter importiert als auch Ersparnisse im Ausland angelegt werden. Abgesehen davon, dass Ersparnisse de facto - unbeschadet aller Globalisierung - nach wie vor überwiegend im Inland angelegt werden (vgl. Coakley et al. I998), ${ }^{15}$ wäre das jedoch nur dann richtig, wenn die Alterung der Bevölkerung bloß ein Land betreffen würde; tatsächlich sind davon jedoch alle Industriestaaten betroffen, wenn auch in leicht unterschiedlichem Ausmaß. ${ }^{16}$ Simulationen (mittels der durchaus unterschiedlichen Modelle) von BörschSupan et al. (2003) und von Fehr/Halder (2004: 29) zeigen dem gemäß auch nur geringe Unterschiede zwischen den Simulationen für eine geschlossene und eine offene Wirtschaft.

\section{Das unverzichtbare Umlageverfahren}

Da sich die Altersversorgung einer schrumpfenden Bevölkerung somit primär als ein Problem der Güterversorgung darstellt, nämlich der Aufteilung einer relativ geringeren Produktion auf weniger Arbeitende und mehr Pensionist|inn|en, und das Finanzierungsproblem demgegenüber sekundär bleibt, geht die gegenwärtige Diskussion über die Finanzierung der Altersversorgung im Wege eines Umlage- oder eines Kapitalstockverfahrens an den wirklichen Problemen vorbei. Den derzeit dominierenden Neokonservativismus interessiert jedoch nicht das Verteilungsproblem, sondern die potentiell ersparnis- und damit wachstumssenkende Wirkung des Umlageverfahrens:

»But for all good it has done, social security is severely limiting world saving, investment, and growth. It threatens to bankrupt the young and future generations of some of the world leading nations. It is the chain-letter pay-as-you-go method of financing social security that lies at the heart of the problem." (Kotlikoff 1996: 368)

I4 Die eher populäre Literatur sieht die Gefahr für die Babyboom-Generation »to drown in financial assets. The consequences should be disastrous, not only for the boomer retirement but also for the economic health of the entire population " (Siegel 1998); nach der - allerdings schwachen - historischen Evidenz ist das erheblich übertrieben (vgl. Poterba 200I).

I5 Feldstein / Horioka (1980) fanden, dass die Ersparnisse - entgegen neoklassischen Erwartungen überwiegend im jeweiligen Inland angelegt werden; auch die zunehmende Globalisierung änderte daran wenig. Das Phänomen wird zumeist mit Informationsasymmetrien erklärt (vgl. French/ Poterba 1999). I6 Allein in Afrika sowie in Süd- und Westasien wird die demografische Altersbelastung sinken, nicht jedoch in Ostasien, das noch am ehesten für die Anlage von westeuropäischen Ersparnissen in Frage käme. 
Zusätzlich zu den Vorwürfen, das Umlageverfahren schädige die Spartätigkeit, damit Investitionen und Wachstum, und es benachteilige die Jungen gegenüber den Alten, werden in Europa noch zwei weitere kritische Argumente vorgebracht: Dass das Umlageverfahren unfinanzierbar und überdies gegenüber dem Kapitalstockverfahren relativ ineffizient wäre. Es bringe bloß eine Rendite in Höhe des Wachstums der Lohnsumme (real etwa I,5 Prozent), ${ }^{17}$ wogegen das Kapitalstockverfahren eine Rendite wenigstens in Höhe der Obligationenrendite (etwa vier Prozent) abwerfe (vgl. Wissenschaftlicher Beirat beim Bundesministerium für Wirtschaft 1998, Sinn 2000). Aus allen genannten Gründen müsse das Umlageverfahren ganz oder wenigstens teilweise durch ein Kapitalstockverfahren ersetzt werden, das möglichst privat betrieben wird; Marquart/Peters (1997) sehen es dem gemäß als »collective madness", wenn sich die Bürger|innen für das Umlageverfahren aussprechen, und können diese Entscheidung nur durch das Altern des/der (egoistischen) Medianwählers / Medianwählerin erklären.

Die Einwände gegen das Umlageverfahren gehen nicht bloß an der Funktion einer Sozialversicherung vorbei, sie sind auch empirisch zumindest umstritten oder für die gegenwärtige wirtschaftliche Lage irrelevant:

I. Selbst wenn das Umlageverfahren die Spartätigkeit reduzieren sollte, wäre das für die europäische Situation einer schon über ein Jahrzehnt anhaltenden Phase der Konsumschwäche und der Unterauslastung der Kapazitäten irrelevant; die europäische Wachstumsschwäche hat ganz andere Ursachen als den Mangel an Ersparnissen. Überdies gibt es zwar für die USA gewisse Anzeichen für eine sozialversicherungsbedingte Reduzierung der Spartätigkeit (vgl. Feldstein 1974, Gokhale et al. 1996), und für die USA ist die Sparschwäche auch tatsächlich eine zunehmend problematische Entwicklung, ${ }^{18}$ für Europa konnte jedoch weder ein Verdrängungseffekt (capital replacement) der Umlagefinanzierung nachgewiesen werden (vgl. Mackenzie et al. 1997, Rürup I997 auf Grund der Daten von Hughes 1996), noch gibt es hier eine Sparschwäche.

2. Dem Problem der mangelnden Generationengerechtigkeit, der Benachteiligung der "Jungen «, liegt die in Kapitel zwei diskutierte mangelnde Transferierbarkeit der Güter zwischen den Generationen und die beschränkte Möglichkeit des Ansparens zugrunde; das ist jedoch keine Schwäche, die auf die Umlagefinanzierung beschränkt ist: Auch unter einem Kapitalstockverfahren können Arbeitende wie Pensionist|inn|en nur das

I7 Als Rendite wird dabei die Verzinsung der Einzahlungen verstanden; beim Umlageverfahren sind die Sozialversicherungsbeiträge die Einzahlungen, die Pensionen die Auszahlungen; wenn sich beide (Indexierung) nach der (jeweiligen) Lohnhöhe richten, entspricht die Rendite dem Lohnwachstum. Bei Kapitalstocksystemen entspricht die Rendite dem Kapitalmarktzins (zu dem die Prämien angelegt werden) abzüglich der Verwaltungskosten. Die üblichen Vergleichsrechnungen vernachlässigen nicht bloß die Wirkung zusätzlichen Sparens auf den Zinssatz sondern zumeist auch, dass die Verwaltungs- und Anlagekosten privater Versicherungen auf rund is Prozent der Bruttoprämien (Westerheide 2000) und höher (vgl. Budden 1997, Diamond 1998, Murthi et al. 200I) geschätzt werden.

I8 Nicht zufällig betont Kotlikoff (1996) "severely limiting world saving «, da in den USA tatsächlich ein arges Spardefizit besteht, das durch europäische und asiatische Ersparnisse finanziert wird. 
verbrauchen, was in der jeweiligen Periode produziert wird. Abgesehen davon wäre ein Systemwechsel keinesfalls generationengerecht, weil er die Übergangsgeneration doppelt belastet.

3. Die angebliche Unfinanzierbarkeit des Umlageverfahrens besteht bestenfalls unter den derzeit geltenden Konditionen, mangels Anpassung der Finanzierung und der Leistungen an die künftigen Voraussetzungen; die Behauptung täuscht darüber hinweg, dass die Finanzierung in jedem Fall von den Bürger|inne|n getragen werden muss, in Form von Steuern und Sozialversicherungsbeiträgen beim Umlageverfahren, ${ }^{\text {I9 }}$ von zwangsweisen oder freiwilligen Beiträgen zu Pensionskassen beim Kapitalstockverfahren oder in Form individueller Ersparnisse welcher Art auch immer im Rahmen der so genannten dritten Säule.

4. Was schließlich die Rendite betrifft, sollte zunächst betont werden, dass »es als nicht systemadäquat [erscheint], die gesetzliche Rentenversicherung primär nach der Höhe ihrer Rendite zu beurteilen. [...] Selbst wenn sie als Versicherung im klassischen Sinn angesehen werden könnte, was zweifelhaft erscheint, so ist sie doch als Bestandteil unseres Sozialversicherungssystems ein Eckpfeiler des auf dem Solidaritätsprinzip aufbauenden Sozialstaats und insoweit keineswegs allein unter Renditegesichtspunkten zu sehen.«(Bomsdorf I998: II)

Darüber hinaus übersieht ein naiver Vergleich der Renditen, dass eine Umstellung des gesamten Pensionssystems auf ein Kapitalstockverfahren einen Deckungsstock in der Höhe von mindestens des Dreifachen des BIP ausmachen und vermutlich den gesamten Kapitalbestand der Volkswirtschaft übersteigen würde (Oksanen 200I: I2); ${ }^{20}$ von den damit verbundenen sonstigen Problemen abgesehen würde der Zinssatz unter dem Einfluss dieser Sparlawine wohl außerordentlich stark sinken, sodass die Argumentation mit der höheren Rendite (Effizienz) des Kapitalstockverfahrens schon aus diesem Grund zusammenbricht.

Die Einwände gegen das Umlageverfahren sind somit schon als solche - jedenfalls für Europa - wenig überzeugend; ihre wirkliche Schwäche ist jedoch darin zu sehen, dass sie die Aufgaben und Möglichkeiten eines Systems der kollektiven Altersvorsorge nicht erkennen: Ein Umlageverfahren kann mehr und anderes abdecken als ein Kapitalstockverfahren. Entscheidend ist die Unterscheidung zwischen Risiko und Unsicherheit: Bei Risiken lässt sich (auf Grund des Gesetzes der großen Zahl) die Wahrscheinlichkeitsverteilung der Ergebnisse abschätzen, bei Unsicherheit nicht. Eine (private) Versicherung (Kapitaldeckung) kann daher Risken absichern, nicht jedoch Unsicherheit. Weiters ist zwischen beitragsorientierter (»defined contribution« - DC) und ergebnis- bzw. leistungsorientierter (»defined benefit« - DB) Gestaltung des Systems zu differenzieren: Im ersten Fall erhält der/die Pen-

I9 Dass die Kosten zwischen Staat, Arbeitgeber|inne|n und Arbeitnehmer|inne|n gedrittelt werden, ist eine Illusion: Das staatliche Drittel zahlen die Bürger|innen in Form von Steuern und den überwiegenden Teil des Arbeitgeber|innendrittels in Form von niedrigeren Löhnen.

20 Krupp (1997) stellt die Frage, ob nicht schon der dramatische Anstieg der Aktienkurse in der Vergangenheit wenigstens z.T. auf das rasche Wachstum der Pensionsfonds zurückzuführen ist, die Anlagemöglichkeiten suchen. 
sionist|in - verzinst oder unverzinst - was er/sie angespart hat, im zweiten eine Pension in vorbestimmter Höhe; ersteres ist im Rahmen einer (privaten) Versicherung möglich, letzteres nicht. Zusammengefasst ergibt das folgendes Schema:

\section{Tabelle 3: Alternative Formen der Finanzierung}

\begin{tabular}{l|ll}
\hline & \multicolumn{1}{|c}{ DC, beitragsorientiert } & \multicolumn{1}{c}{ DB, ergebnisorientiert } \\
\hline Umlage & $\begin{array}{l}\text { Persönliche Konten } \\
\text { (Notional defined contribution, } \\
\text { kurz NDC) }\end{array}$ & Konventionelles Sozialversicherungsmodell \\
Kapitaldeckung & Versicherungsmodell & Traditionelles Firmenpensionsmodell \\
\hline
\end{tabular}

Quelle: Eigene Zusammenstellung

Zentral ist, dass das Kapitaldeckungsmodell zwar das Risiko der Lebenserwartung abdecken kann, indem überdurchschnittlich lang Lebende die Pension länger und unterdurchschnittlich lang Lebende kürzer erhalten, damit mehr bzw. weniger als sie angespart haben; aber schon die Pensionshöhe kann das Kapitaldeckungsmodell ex ante nicht garantieren: Diese hängt von der Entwicklung der Verzinsung und sonstigen Einflüssen auf den Kapitalstock während der Ansparperiode ab. ${ }^{21}$ Krieg, galoppierende Inflation oder ähnliche Katastrophen können die Kapitaldeckung vernichten, sodass die Pensionist|inn|en u.U. sogar völlig leer ausgehen; Alier/Vittas (200I) sowie Burtless (2003) haben gezeigt, wie stark sich die historischen Kapitalmarktschwankungen auf die US-amerikanischen Pensionen auswirkten. Selbst die aus historischer Sicht eher unbedeutende - weil allein auf Zurückführung einer spekulativen Überbewertung beruhende - jüngste Aktienbaisse in den USA hat die Pensionssysteme und noch mehr die Pensionist|inn|en empfindlich getroffen. ${ }^{22}$ Die Umlagefinanzierung kann hingegen die großen Risiken von Krisen (vor allem des Kapitalmarkts), von Kriegen oder galoppierender Inflation zumindest finanziell abdecken, und insoweit die jeweiligen Verluste auf die ganze Bevölkerung aufteilen. Insofern handeln die Bürger|innen - entgegen den Vorstellungen von Marquart/ Peters (I997) - durchaus rational, wenn sie sich für das Umlageverfahren aussprechen; die wirklich großen Unsicherheitselemente bewegen sie, nicht zu Unrecht, mehr als z.T. hypothetische und unsichere Effizienzvorteile - jedenfalls solange die Kapitalverluste aus einer Weltwirtschaftskrise und zwei Weltkriegen noch bewusst sind und durch laufende kleinere Kapitalmarktkrisen weiterhin bewusst gehalten werden.

Das Umlageverfahren gewinnt im Bewusstsein der Öffentlichkeit sogar an Bedeutung, weil früher ergebnisorientierte (DB) Pensionen - die besseren Versicherungsschutz bieten -

\footnotetext{
2I Dazu können noch Managementfehler der öffentlichen oder privaten Verwalter|innen kommen. 22 Als besonders problematisch hat sich die von der Weltbank forcierte Umstellung der lateinamerikanischen Pensionssysteme (vor allem in Argentinien und Chile) auf überwiegende oder gar ausschließliche Kapitaldeckung herausgestellt; die Pensionskrise dieser Länder hat inzwischen selbst die Weltbank zu einem Umdenken veranlasst.
} 
wenigstens in Form von Firmenpensionen für besser Verdienende eine nicht unerhebliche Rolle spielten. Mit deren zügigem Abbau bzw. dem Umbau der so genannten zweiten Säule auf Pensionskassen (= Beitragsorientierung, DC) werden Zins- wie Altersrisiko zunehmend auf die Versicherten verlagert. ${ }^{23}$ Diese Verlagerung, durch die Pensionist|inn|en zu einem "gesamtwirtschaftlichen Risiko-Stoßdämpfer« werden, besorgt inzwischen selbst den Internationalen Währungsfonds (2005), der nicht bloß bessere Aufklärung der Bürger|innen über die Risiken verlangt, sondern den Staaten auch empfiehlt, für die Altersicherung besser geeignete Anlageformen zu schaffen. ${ }^{24}$ Eine Tendenz, Risiken auf die Pensionist|inn|en abzuwälzen, zeigt sich in den zunehmenden Versuchen, DC-Elemente in Form von "persönlichen Konten « in das Umlageverfahren einzubauen (notional defined contribution, NDC), wie das in Schweden bereits erfolgt ist (vgl. Williamson 200I) und womit auch anderswo, darunter in Österreich, experimentiert wird. Dabei wird eine virtuelle Beziehung zwischen Einzahlungen und Anwartschaft hergestellt; wieweit dabei die Vorteile des Umlageverfahrens erhalten bleiben bzw. Risiken auf die Versicherten abgewälzt werden, hängt von der spezifischen Ausgestaltung ab, vor allem der Art der Verzinsung (Lohnsummen-, Inflationsoder Kapitalmarktzins-abhängig) und der Höhe der (eventuellen) Mindestpension.

Abschließend muss noch ein zentraler ordnungspolitischer Vorteil der Umlagefinanzierung erwähnt werden: Schon zu Beginn dieses Abschnitts wurde darauf hingewiesen, dass der Kapitalstock bei einem Kapitalstockverfahren rund das Dreifache des BIP ausmachen und somit etwa dem gesamten Kapitalbestand der Volkswirtschaft entsprechen würde. Eine solche enorme Kapitalakkumulation müsste zwangsläufig zu der für die USA typischen Dominanz der Kapitalinteressen und zu der problematischen Steuerung der gesamten Wirtschaft über den Kapitalmarkt führen. ${ }^{25}$

23 Die europäische Diskussion um die Finanzierung des eigenen Sozialsystems übersieht, dass sich auch das US-amerikanische in - wenn auch anderen - Schwierigkeiten befindet. Dort gab es immer schon drei Säulen, von denen die Sozialversicherung - entgegen landläufiger Meinung - die bedeutendste ist: Diese Säule trägt zu 38 Prozent zu den Einkommen der über 65-Jährigen bei, Kapitalerträge zu I8 Prozent, Firmen- und Staatspensionen zu je neun Prozent und Arbeitseinkünfte zu 23 Prozent, was bei einer Sozialversicherungsrente, die im Durchschnitt bloß rund 90o US-\$ pro Monat ausmacht, nicht weiter verwunderlich ist (vgl. Munnell et al. 2004); die Sozialversicherung erzielt derzeit Überschüsse und erwartet Defizite frühestens in einigen Jahrzehnten (vgl. knowledge@wharton 2005). Die zweite Säule verliert an Bedeutung: die Zahl der Firmen, die betriebliche Altersvorsorge anbieten, ist in den letzten Jahren dramatisch geschrumpft, und die verbleibenden sind von Renten auf Sparpläne umgestiegen (vgl. Clark/Mitchell 2004), wodurch ein Großteil der Risiken auf die Versicherten verlagert wurde; der Rückzug der Firmen hat die staatliche PBGC (die die Firmenpensionen absichert) in erhebliche Finanzierungsprobleme gebracht (vgl. knowledge@wharton 2004; Mitchell o. J.). Die dritte Säule, das individuelle Sparen, reicht vor allem bei den Ärmeren nicht aus, den Lebensstandard angemessen aufrecht zu erhalten (vgl. Mitchell 1998).

24 Etwa inflationsindexierte Staatsanleihen oder »Langlebigkeits-Bonds«, deren Auszahlungssumme bei geringerer Sterblichkeit steigt.

25 Die Steuerung des US-amerikanischen Wirtschaftssystems erfolgt ausschließlich durch den Kapitalmarkt, also letztlich durch die von Finanzintermediären vertretenen Aktionärinnen- und Aktionärsinteressen (»shareholder value»). Selbst die Unternehmer|innen und Manager|innen haben unter 


\section{Die Erwartungen der Betroffenen}

Die Analyse zeigt, dass die Finanzierung guter, verglichen zu heute sogar besserer Lebensqualität im Alter auch unter der künftigen demografischen Struktur möglich ist; die Verteilung des künftigen Produktionsvolumens auf Arbeitende und Ruheständler|innen bedarf allerdings einer Anpassung durch Verbreiterung der Finanzierungsbasis, Erhöhung der Beitragszahlungen, Erhöhung des Pensionsantrittsalters oder Senkung der Ersatzquote. Das höhere Brutto-Nationalprodukt macht es bei fairer Lösung des Aufteilungsproblems dennoch möglich, alle Beteiligten in Zukunft auch netto besser zu stellen als heute. Die neokonservative Ideologie setzt jedoch auf einen Abbau des Sozialstaats und eine Ablösung des Umlageprinzips durch zumindest teilweise Fundierung und Privatisierung der Altersversorgung (vgl. Kotlikoff 1996) ${ }^{26}$ Sie erkennt nicht, dass der Sozialstaat die Verunsicherung der Bürger abbaut, die in einer Periode raschen Wandels ohnedies groß ist (vgl. Tichy 2004a, 2005b), und damit das Wohlbefinden der Bürger|innen - über die bloß physische Absicherung hinaus - erhöht; ${ }^{27}$ damit schafft der Sozialstaat zugleich wichtige Voraussetzungen für eine produktivitätssteigernde Spezialisierung der Arbeitskräfte (vgl. Tichy 2003, Auer et al. 2004) und für sozialen Frieden.

Wie aber sehen die Bürger|innen die Zukunft der Alterssicherung? Was wollen sie? Die Europäer|innen haben - anders als die US-Amerikaner|innen - ein hohes Interesse an Umverteilung und sozialer Absicherung, und ihr Bedarf danach steigt mit steigendem Einkommen weiter (vgl. Botero et al. 2003) ${ }^{28}$ In einer Spezial-Eurobarometer Umfrage aus dem Jahr 1992 (vgl. Ferrera 1993) bekannte sich eine überwiegende Mehrheit der befragten EU-Bürger|innen zur Beibehaltung des Sozialstaats in seiner gegenwärtigen Form, auch um den Preis höherer Beiträge oder Steuern; die Zufriedenheit mit der gegenwärtigen Form der sozialen Absicherung erwies sich in den nordischen Staaten größer als im Süden, wo -

diesen Voraussetzungen bloß beschränkten Entscheidungsspielraum - sie müssen sich so verhalten, dass der Kurs ihrer Aktien steigt, damit die Fonds ihre Aktien kaufen -, vom mangelnden Einfluss der sonstigen stakeholder nicht zu reden. Das System erzwingt sogar deren schwache Stellung: Denn auf jede Bedrohung des Umsatzes oder Gewinns - welchen Ursprungs auch immer - muss der/die Unternehmer|in/Manager|in sofort reagieren, um den Aktienkurs halten zu können. Mangels anderer Alternativen kann die Reaktion bloß in Entlassungen liegen (vgl. Tichy 2005c); ein voll flexibler Arbeitsmarkt, unsichere Arbeitsplätze und Mangel an Mitbestimmung sind daher mit dem System der Kapitalmarktsteuerung zwangsläufig und untrennbar verbunden.

26 Es überrascht daher wenig, dass nur 29 Prozent der Österreicher|innen die jüngste Pensionsreform als Sicherung der Pensionen einschätzen, 63 Prozent hingegen als reine "Geldbeschaffungsaktion«. Am ehesten sehen Selbständige (42 Prozent) und über 6o-Jährige die Reform als Zukunftssicherung, doch sind die Unterschiede zu den unter 29-Jährigen (20 Prozent) kleiner als zumeist angenommen (vgl. Palme 2003).

27 Der Sozialstaat dient der "universal reduction in the uncertainty faced by individuals« (Havemann I985).

28 "Here the results are clear: richer countries have more extensive social security protection in all areas, including old age, disability and death, sickness, and unemployment." (Botero et al. 2003: I8) . 
zu Recht - eher Reformbedarf gesehen wird (ebd.: 37). Die EU-weite Evidenz des SpezialEurobarometers 1992 wird durch eine neuere Umfrage (März 200o) in Frankreich, Italien, Spanien sowie (getrennt) in Ost- und Westdeutschland (je I.ooo Personen) voll bestätigt (vgl. Boeri et al. 200I): Abermals wünscht die überwiegende Mehrzahl der Befragten keine Reduktion des Sozialstaats und wäre sogar bereit, für Sozialleistungen mehr zu bezahlen (ebd.: Io). Auch zwei Drittel (GfK 2002) bis vier Fünftel (vgl. Palme 2003) der Österreicher|innen sehen den Sozialstaat als sehr wichtig an, Selbständige sogar in überdurchschnittlichem Maße; von den einzelnen sozialen Bereichen halten 84 Prozent das Pensionswesen für sehr wichtig oder wichtig - nach dem Gesundheitswesen (92 Prozent), dem Schulwesen (88 Prozent) und der Pflegesicherung (86 Prozent), und zwar weitgehend unabhängig vom Alter (ebd.). 62 Prozent der Österreicher|innen sind mit der Entwicklung des Sozialstaats sehr zufrieden oder zufrieden, ${ }^{29}$ drei Viertel sehen das Pensionssystem allerdings bedroht, und zwar die Jüngeren stärker (bis 29 Jahre 85 Prozent) als die Älteren (über 65 Jahre 62 Prozent) (vgl. ebd.).

Eine EU-weite Umfrage im Herbst 200I (European Research Group 2004: Question 56.I und Question 62.9) bestätigt den nachhaltigen Wunsch nach staatlicher Sicherung des Alters: Drei Viertel der Österreicher|innen wie der EU-Bürger|innen befürworten einen Zwang zur Pensionsversicherung, rund 85 Prozent wollen - weitgehend unabhängig vom Alter - ein Pensionssystem, das die Beibehaltung eines adäquaten Lebensstandards ermöglicht (ebd.: Question 62.5); gleichfalls 85 Prozent der Österreicher|innen (und sogar 92 Prozent der EU-Bürger|innen) verlangen eine garantierte Mindestpension (ebd.: Question 62.4). Für die von der Politik forcierten Pensionskonten (notional defined contribution, NDC) treten bloß 46 Prozent der Österreicher|innen (56 Prozent der EU-Bürger|innen) ein, in Verbindung mit einer staatlichen Mindestpension jedoch 8o Prozent (82 Prozent). Aussteigen aus dem Pensionsversicherungssystem (»opting out «) möchte nur ein kleiner Teil (Boeri et al. 200I: 3I f.), obwohl die Mehrzahl eine Krise des Pensionssystems erwartet (ebd.: 43). ${ }^{30}$

Was die Pensionshöhe betrifft, betrachten Is Prozent der berufstätigen ${ }^{31}$ Österreicher|innen (EU I8 Prozent) 50 bis 70 Prozent ihres Aktiv-Nettoeinkommens als ausreichend, knapp 60 Prozent (53 Prozent) wollen jedoch mehr (Spezial-Eurobarometer 56.I: Question 5I), wobei sich vor allem Akademiker|innen und Angestellte sowie Männer als überdurchschnittlich anspruchsvoll erweisen. Sie glauben, das auch zu bekommen, denn zwei Drittel aller berufstätigen Österreicher|innen (62 Prozent EU) erwarten in der Pension keine finanziellen Probleme (ebd.: Question 55); dem entspricht, dass 82 Prozent aller Österreicher|innen (EU

30 Das ist keineswegs irrational: Schon vor zwei Jahrzehnten hat Katzenstein (1985: 47) darauf hingewiesen, dass höhere Risiken einen Bedarf an staatlichen Absicherungsstrategien auslösen, und Rodrik (1998) wie Agell (1999) haben das jüngst empirisch bestätigt. Rodrik konnte auch nachweisen, dass diese Absicherungsstrategien in II 9 der 147 von ihm untersuchten Ländern signifikant stabilisierend wirkten. Soziale Absicherung hilft somit nicht bloß dem Einzelnen, sie ist auch gesamtwirtschaftlich produktiv - nicht kontraproduktiv, wie die herrschende Wirtschaftspolitik behauptet -, von ihren positiven gesellschaftlichen Folgen ganz abgesehen.

3I Genauer: der "non-pensioners". 
75 Prozent) ihren gegenwärtigen Lebensstandard tatsächlich als zumindest durchschnittlich empfinden, mit relativ geringen Unterschieden zwischen Berufstätigen und Pensionist|inn|en; ${ }^{32}$ zwei Drittel der Pensionist|inn|en erwarten keine Verschlechterung in den kommenden fünf bis zehn Jahren (ebd.: Question 6o). Sollte es jedoch Probleme geben, ziehen zwei Drittel der Österreicher|innen wie der EU-Bürger|innen (drei Viertel der Pensionist|inn|en) höhere Beiträge einer Kürzung der Pensionen vor (ebd.: Question 67.I). ${ }^{33}$ Aus neokonservativer Sicht mögen diese Vorstellungen der Bürger|innen naiv, als Verdrängung der drohenden Finanzierungsprobleme erscheinen, und sie widersprechen den Elaboraten einer aufgeregten Medienszene (vgl. Tichy 2004b) ebenso wie den Behauptungen und Aktionen der Politik der meisten Staaten; mit den Schätzungen im ersten Abschnitt dieser Arbeit ist der Optimismus der Befragten jedoch keineswegs inkompatibel.

Etwas anders erweist sich die Lage bezüglich des Pensionsantrittsalters. Zwar werden die Beschäftigten derzeit i. a. etwas früher in Pension geschickt als sie intendieren - in Österreich mit 57,2 statt mit den von den Bürger|inne|n präferierten 59 Jahren (EU 59,4 statt $60,3)$, doch im Allgemeinen will man in der EU derzeit nicht nennenswert länger arbeiten als bis zum 6o. Lebensjahr. ${ }^{34}$ Bloß ein knappes Viertel der österreichischen wie der EU-Bürger|innen würde einer Heraufsetzung des Pensionsantrittsalters deutlich und ein weiteres Zehntel zögernd zustimmen (ebd.: Question 67.3). Allerdings mag das z.T. an der ungeschickten Fragestellung liegen, da - anders als bei der Beitragserhöhung - nicht nach der Alternative Pensionskürzung versus Beitragserhöhung gefragt wurde. ${ }^{35}$ Vor allem die Österreicher|innen (77 Prozent gegen 44 Prozent im EU-Durchschnitt) erwarten gemäß einer früheren Umfrage (European Research Group 200I: Question I7), dass das Pensionsantrittsalter in den nächsten fünf bis zehn Jahren steigen wird; ${ }^{36}$ allerdings liegt es hierzulande auch besonders niedrig. Drei Viertel der Österreicher|innen wären bereit, zur Sicherung des Umlageverfahrens länger zu arbeiten (Zöchling 2003: 28), doch gibt es auch hier keine Angaben, wie lange. Insgesamt bleibt der Eindruck, dass es gegen eine Erhöhung des Antrittsalters über das Alter von 6o Jahren hinaus derzeit doch einen gewissen Widerstand gibt.

\footnotetext{
32 Das entspricht auch den Ergebnissen der Armutsforschung (vgl. Tichy 2005a).

33 Die Bereitschaft zu Eigenleistungen ist in Österreich für Pensionen ( 54 Prozent der Befragten) merklich höher als für Hochschulgebühren (48 Prozent) oder Selbstbehalte im Gesundheitswesen (vgl. Palme 2003). Gegen Beitragserhöhungen wendet sich vor allem die Wirtschaft, die daran anteilsmäßig beteiligt ist.

34 Die Spanne reicht von 63 und 62 Jahren in Dänemark und Spanien, bis zu 58 und 57 Jahren in Belgien und Luxemburg.

35 Gefragt wurde nach der Zustimmung zu dem Vorschlag: "The age of retirement should be raised so that people work longer and therefore spend less time in retirement".

36 Sechs Prozent (I6 Prozent) glauben, dass es gleich bleiben wird, II Prozent (32 Prozent) erwarten eine Senkung.
} 


\section{Elemente einer Reform}

Eine nachhaltige Pensionsreform wird davon ausgehen müssen, dass die Gruppe der Pensionist|inn|en in Zukunft noch erheblich stärker differenziert sein wird als heute. Einer der Gründe dafür ist die steigende Lebenserwartung: Noch in den I970er Jahren ging man wie Abbildung 3 zeigt - mit 60 Jahren in Pension und starb mit 70 Jahren; schon rein altersmäßig war die Gruppe der Ruheständler|innen somit recht homogen. Wenn die Lebenserwartung im Jahr 2040 auf etwa 85 Jahre geschätzt wird, verdoppelt sich die Altersspanne der Pensionist|inn|en selbst bei Erhöhung des Pensionsantrittsalters auf etwa 20 Jahre. Die Pensionist|inn|en werden dann altersmäßig gleichermaßen differenziert sein wie die Gruppe der Jugendlichen zwischen zehn und 30 Jahren. Das bedeutet, dass sie sich auch physisch und psychisch erheblich stärker unterscheiden werden als heute, unterschiedlich aktiv und unterschiedlich bereit und fähig zu arbeiten.

Abbildung 3: Länge der einzelnen Lebensphasen*

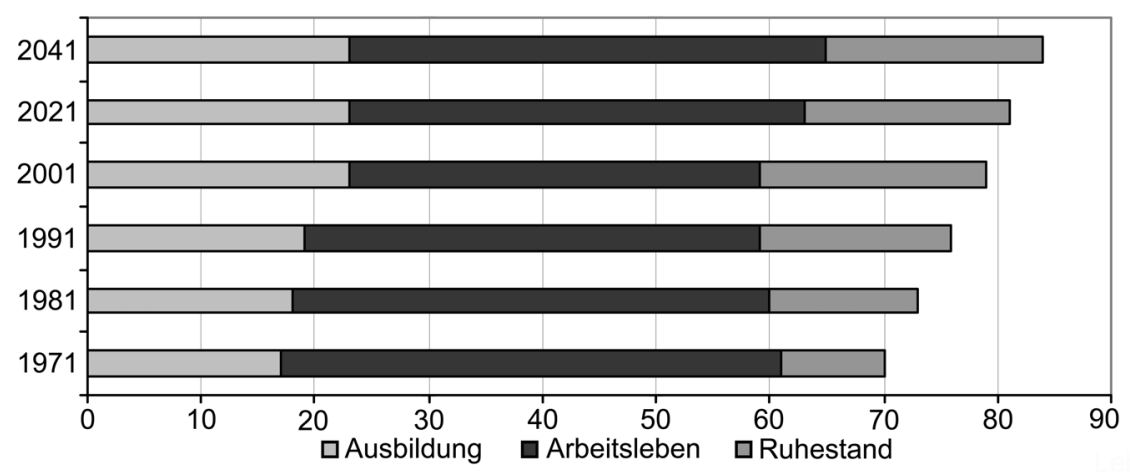

* Die Daten der Übersicht wurden von Statistik Austria mittels Erwerbsquoten berechnet; das Ergebnis ist konsistent mit den von Guger und Mayrhuber (2004: 827) angegebenen Werten des Pensionsantrittsalters von 57,6 (1999) bzw. 58,2 (2003) Jahren.

Quelle: 197I-200I Statistik Austria, berechnet mittels Erwerbsquoten, Unter-bzw. Obergrenze von "Arbeitsleben" bei 70 Prozent bzw. 30 Prozent der Erwerbsquote; Obergrenze von Ruhestand: Lebenserwartung bei der Geburt. 202I-204I: Annahmen des Autors gemäß Abschnitt I.I

Überdies wird sich ihre materielle Lage aus verschiedenen Gründen stärker differenzieren: Die problematische Arbeitsmarktlage der jüngeren Vergangenheit, die wohl noch einige Zeit anhalten wird, wird für manche eher unzureichende Pensionen bedeuten; Arbeitnehmer|innen mit höherem Einkommen und kontinuierlichen Erwerbskarrieren werden ihre ohnedies höheren Pensionen durch zusätzliche Ersparnisse aufbessern können. Vor allem aber darf nicht übersehen werden, dass etliche - als Folge der höheren Lebenserwartung ihrer Vorfahren - erst im Ruhestand oder knapp zuvor deren Vermögen erben werden.

Für eine Pensionsreform bedeutet die zunehmende Differenzierung der Gruppe der Pensionist|inn|en zweierlei: Erstens, dass das Antrittsalter variabel gestaltet werden sollte, 
mit entsprechenden $\mathrm{Zu}$ - und Abschlägen betreffend die Höhe der Pension oder auch betreffend die Höhe der Beiträge. Zweitens, dass das Steuersystem auf die neue materielle Differenzierung im Alter reagieren muss, indem Erbschaften und Kapitalerträge stärker besteuert werden; denn das - und nicht so sehr eine Kürzung der Normalpensionen - entspräche der von den Bürger|inne|n so sehr gewünschten fairen Heranziehung der »Alten « zur Intergenerationenverteilung, da die Besteuerung in die Periode der gütermäßigen Belastung fällt. ${ }^{37}$

Zusätzlich zu der Berücksichtigung der markanten Differenzierung der Struktur und der Leistungsfähigkeit der kommenden Pensionist|inn|engeneration wird eine nachhaltige Reform zweitens zwischen den verschiedenen »Umverteilungstöpfen « umschichten müssen. Die Analyse hat klar gezeigt, dass in Zukunft weniger Kinder, Arbeitslose und Frühpensionist|inn|en erhalten werden müssen, aber mehr Pensionist|inn|en. Das bedeutet, dass ceteris paribus weniger Mittel für Familienförderung und Arbeitslosenunterstützung benötigt werden, mehr hingegen für Altersversorgung. Die wohl unvermeidlichen Beitragserhöhungen im Bereich der Altersicherung können daher durch Beitragssenkungen in den anderen Bereichen zumindest teilweise kompensiert werden.

Drittens sollte sich die Politik - aber auch die wissenschaftliche Literatur - über die vieldiskutierte Verteilungsgerechtigkeit zwischen den Generationen hinaus doch sehr viel intensiver mit der Verteilungsgerechtigkeit innerhalb der jeweiligen Generation beschäftigen. ${ }^{38}$ Das Problem geht natürlich über die Frage der Altersfinanzierung weit hinaus, sodass hier bloß die dafür relevanten Aspekte erwähnt werden können. Das betrifft vor allem die Konzentration auf die Arbeitseinkommen als Finanzierungsbasis, die über die Verteilungsproblematik hinaus auch das Problem einer Verteuerung des Produktionsfaktors Arbeit bedeutet. Es betrifft weiters die unterschiedliche Besteuerung der Alterseinkommen (Pension, Kapitaleinkommen, Erbschaften), die de facto-Benachteiligung von Frauen und die Gleichbehandlung von Personen mit und ohne Kinder. ${ }^{39}$ Es muss allerdings nochmals darauf hingewiesen

37 8o Prozent der Befragten halten es für ungerecht, »dass die heutigen Pensionisten mit hoher Pension keinen Solidarbeitrag leisten müssen.« (vgl. Palme 2003)

38 Da es schwer fällt, die fast völlige Vernachlässigung dieser Frage durch die öffentliche - und wissenschaftliche - Diskussion dem Zufall zuzuschreiben, liegt eine ideologische Erklärung nahe: Dem neokonservativen Mainstream geht es - angebotstheoretisch - primär nicht so sehr um Generationengerechtigkeit sondern vielmehr um Kapitalbildung und Stärkung der Finanzmärkte.

39 Zwar tragen Singles und Ehepaare ohne Kinder im Wege des Familienlastenausgleichs zur Finanzierung der Kosten der Kinder bei; sie können sich aber auch intensiver um ihre Karriere kümmern, sind dem gemäß weniger häufig arbeitslos und erleiden weniger Unterbrechungen ihres Berufslebens. Dem zufolge haben sie nicht bloß höhere Einkommen (und geringere Ausgaben), sondern auch höhere Pensionen. Kinderlose müssen aber gütermäßig genau so wie Eltern von einer künftigen Generation versorgt werden; insofern sollte intensiver diskutiert werden, wie weit sie auch durch höhere Pensionsbeiträge zu einer fairen Verteilung innerhalb und zwischen den Generationen beitragen könnten. In Deutschland hat das Bundesverfassungsgericht 200I gleiche Beitragssätze für Personen mit und ohne Kinder (in der Pflegeversicherung) als mit dem Gleichheitsgrundsatz unvereinbar abgelehnt. In Österreich stellte ein Institut der Wirtschaftskammer einen derartigen Vorschlag zur Diskussion; die Wirtschaftskammer distanzierte sich jedoch sogleich davon, und Gewerkschaftsvertreter|innen 
werden, dass Umverteilung (i.w. S.) nur zur Linderung des finanziellen, nicht aber zu der desin diesem Beitrag für wichtiger gehaltenen - realen Pensionsproblems beitragen kann.

Ein viertes, eher problematisches, aber überaus wichtiges Element einer nachhaltigen Pensionsreform ist die Erhöhung des Pensionsantrittsalters, problematisch deswegen, weil die Umfragen derzeit vermuten lassen, dass die Bürger|innen eher zu einer Erhöhung der Beiträge bereit wären. Drei Gründe sprechen dennoch dafür, sich mit dieser Frage ernsthaft zu beschäftigen: Erstens, dass - wie bereits erwähnt - die Fragestellung des Eurobarometers in diesem Punkt besonders ungeschickt war und das Ergebnis daher nicht verlässlich ist. Zweitens, dass auf diesem Gebiet Ansteckungseffekte eine besondere Rolle spielen: Wenn die Bekannten aus derselben Altersgruppe, Schulkollegen etc. bereits alle in Pension sind, wenn man sich am Arbeitsplatz geradezu als Fossil fühlt und die Vorstellungen der Jüngeren gemeinsam mit dem technischen und organisatorischen Fortschritt zu anderen Arbeitsabläufen führen, steigert das sicherlich das Bedürfnis aufzuhören. ${ }^{40}$ Drittens ist das Pensionsantrittsalter - vor allem in Österreich - besonders niedrig: Gerade noch die Hälfte der 55- bis 60-Jährigen und ein Achtel der 60- bis 65-Jährigen stand 2002 noch in Arbeit, gegenüber zwei Drittel der 55- bis 64-jährigen Norweger|innen, Schweizer|innen und Schwed)inn|en und immerhin zwei Fünftel der US-Amerikaner|innen und Dän|inn|en. Längere Beschäftigung wäre jedoch eine besonders effiziente Lösung des Pensionsdilemmas, da sie die finanziellen wie die realen Probleme zugleich lösen könnte; sie ist daher der von den Bürger|inne|n in den Umfragen offenbar präferierten Beitragserhöhung gesamtwirtschaftlich und gesamtgesellschaftlich weitaus vorzuziehen: Der Bedarf an Arbeitskräften wird durch die Schrumpfung der Bevölkerung im erwerbsfähigen Alter um rund ein Siebentel drängend sein (Tichy I999: 224 f.), und eine Hinaufsetzung des Pensionsantrittsalters um ein Jahr ermöglicht es, die Beiträge um I, 5 Prozentpunkte weniger stark zu erhöhen. Allerdings bedarf eine solche Lösung eines umfangreichen Begleitprogramms der betrieblichen und überbetrieblichen Schulung und Weiterbildung und einer laufenden Anpassung der Qualifikation auch der älteren Arbeitskräfte an die sich wandelnden Bedürfnisse.

\section{Literatur}

Agell, Jonas (1999): On the Benefits of Rigid Labour Markets: Norms, Market Failures, and Social Insurance, in: Economic Journal, Jg. I09, S. I43-164

Alier, Max/Vittas Dimitri (200I): Personal Pension Plans and Stock Market Volatility, in: Robert Holzmann/Joseph E. Stiglitz (Hg.), New Ideas About Old Age Security, Washington: The World Bank, S. 39I-423

\footnotetext{
betonten, dass man die Kinderlosen nicht »bestrafen« dürfe, eine Reaktion, die an der Fairness-Problematik vorbeigeht.

40 Dazu mag die Angst kommen, früher oder später gekündigt zu werden, was psychologisch schlimmer ist als ein selbstbestimmter Pensionsantritt, oder der Vorwurf, einem/einer Jungen den Arbeitsplatz wegzunehmen.
} 
Auer, Peter et al. (2004): Is a Stable Workforce Good for the Economy? Insights into the Tenureproductivity-employment Relationship, mimeo

Barr, Nicholas (200I): The Welfare State as a Piggy bank: Information, Risk, Uncertainty, and the Role of the State, Oxford/NewYork: Oxford University Press

Boeri, Tito et al. (20or): Would You Like to Shrink the Welfare State? A Survey of European Citizens, in: Economic Policy, Jg. 16, Nr. 32, S. 7-50

Bomsdorf, Eckart (1998): Die Renten sichern - Ein Weg aus der Rentenkrise, Studie zur Zukunft der gesetzlichen Rentenversicherung, 2. Auflage, Köln

Börsch-Supan, Axel et al. (2003): Aging, Pension Reform, and Capital Flows: A Multi-country Simulation Model, mimeo

Botero, Juan et al. (2003): The Regulation of Labor, NBER Working Paper Nr. 9756, Cambridge, Mass.: National Bureau of Economic Research

Budden, Robert (1997): Money Down the Drain, Money Management, Nr. I, S. 46-53

Burtless, Gary (2003): What To Do About the Risk of Individual Account Pensions? Evidence from Industrial Countries, mimeo

Bütler, Monika / Kirchsteiger, Georg (1999): Aging Anxiety: Much Ado About nothing?, CentER Discussion Paper Nr. 9937

Clark, Robert L. / Mitchell, Olivia S. (2004): Changing the Retirement Paradigm, Pension Research Council Working Paper Nr. 2004-I8, Philadelphia: Wharton School

Coakley, Jerry et al. (1998): The Feldstein-Horioka Puzzle and Capital Mobility: A Review, in: International Journal of Finance \& Economics, Jg. 3, Nr. 2, S. 169-188

Diamond, Peter, A. (1998): The Economics of Social Security Reform, in: R. Douglas Arnold et al. (Hg.), Framing the Social Security Debate: Values, Politics, and Economics, Washington: Brookings

European Research Group (200I): The Social Situation in the European Union, Eurobarometer Nr. 54.2, Brüssel: European Commission

European Research Group (2004): The future of pension systems, Special Eurobarometer Nr. 56.I, Brüssel: European Commission

Fehr, Hans/Halder, Gitta (2004): Alternde Bevölkerung, öffentliche Budgets und intergenerative Wohlfahrt, in: Genser, Bernd (Hg.), Haushaltsdisziplin und öffentliche Verschuldung, Berlin (i.E.)

Feldstein, Martin/ Horioka, Charles (1980): Domestic Savings and International Capital Flows, in: Economic Journal, Jg. 90, S. 314-329

Feldstein, Martin (1974): Social Security, Induced Retirement, and Aggregate Capital Accumulation, in: Journal of Political Economy, Jg. 82, Nr. 5, S. $905-926$

Ferrera, Maurizio (1993): EC Citizens and Social Protection. Main Results From an Eurobarometer Survey, Brüssel: European Commission

Fischermann, Thomas (2005a): Bereit zum großen Schlag, in: DIE ZEIT vom I3.0I.2005, S. 2I

Fischermann, Thomas (2005b): Der Zorn des Gouvernators, in: DIE ZEIT vom 13.0I.2005, S. 2 I

Fischermann, Thomas (2000c): Bushs »Blitzkrieg«, in: DIE ZEIT vom 23.03.2005, S. 28

French, Kenneth R. / Poterba, James. M. (1999): Investor Diversification and International Equity Markets, in: American Economic Review Papers and Proceedings, Jg. 8I, Nr. 2), S. 222-226 
Gesellschaft für Sozialforschung (GfK) (2002): Ergebnis-Highlights der Lifestyle-Studie 2002, mimeo

Gokhale, Jagadeesh et al. (1996): Understanding the Postwar Decline in U.S. Saving: A Cohort Analysis, in: Brookings Papers on Economic Activity, Nr. I, S. 315-407

Guger, Alois/Mayrhuber, Christine (2004): Angleichung des Pensionsalters der Frauen an jenes der Männer bis 2030, in: Monatsberichte des Österreichischen Instituts für Wirtschaftsforschung, Jg. 77, Nr. II, S. 825-835

Havemann, Robert H. (1985): Does the Welfare State Increase Welfare?, Inaugural Lecture of the Tinbergen Chair, Leiden: H.E. Stenfert Koese

Hughes, Gerard (1996): Pension Financing, the Substitution Effect and National Savings, Paper for the International Conference on Pensions in the European Union

International Monetary Fund (2004): World Economic Outlook, Washington: IMF

Katzenstein, Peter (1985): Small States in World Markets: Industrial Policy in Europe, Ithaka, NY: Cornell University Press

knowledge@wharton (2004): Underfunded Pensions: Causes, Cures and Questions, http:// knowledge. wharton.upenn.edu/index.cfm? fa=viewArticle \&id $=824$

knowledge@wharton (2005): Is Social Security in Trouble? Depends on Whom You Ask, http:// knowledge.wharton.upenn.edu/article/ır2o.cfm

Kommission der Europäischen Gemeinschaften (2005): Mitteilung der Kommission. Grünbuch "Angesichts des demografischen Wandels - eine neue Solidarität zwischen den Generationen«, Brüssel: Europäische Kommission

Kotlikoff, Laurence J. (1996): Privatizing Social Security at Home and Abroad, in: American Economic Review, Jg. 86, Nr. 2, S. 368-372

Krupp, Hans-Jürgen (1997): Makroökonomische Perspektiven einer Teilkapitaldeckung der Rentenversicherung, in: Wirtschaftsdienst, Jg. 77, Nr. 4, S. 203-2II

Mackenzie, George et al. (1997): Pension Regimes and Saving, IMF Occasional Paper Nr. I53, Washington

Marquart, Marko / Peters Wolfgang (1997): Collective Madness. How Ageing Influences Majority Voting on Public Pensions, Sonderforschungsbereich 303 Discussion Paper Nr. A-548, Universität Bonn

Mitchell, Olivia S. (1998): Developments in Pensions, in: NBER-Reporter Spring 1998

Mitchell, Olivia S. (o. J.): Testimony, http://aging.senate.gov/events/hrı5os.htm

Munnell, Alicia et al. (2004): An Update of Employer-sponsored Pensions, Center for Retirement Research at Boston College

Murthi, Mamta et al. (200I): Administrative Costs Under a Decentralized Approach to Individual Accounts: Lessons From the United Kingdom, in: Holzmann, Robert/Stiglitz, Joseph E. (Hg.), New Ideas About Old Age Security, Washington: The World Bank, S. 308-335

Oksanen, Heikki (20or): Pension Reforms for Sustainability and Fairness, in: CESifo Forum, Jg. 2, Nr. 4, S. I2-I8

Palme, Imma (2003): Einstellung zum Sozialstaat, Folien, Wien: IFES

Poterba, James. M. (200I): Demographic Structure and Asset Returns, in: Review of Economics and Statistics, Jg. 83, Nr. 4, S. 565-584 
Rodrik, Dani (1998): Why do More Open Economies Have Bigger Governments?, in: Journal of Political Economy, Jg. I06, Nr. 5, S. 997-1032

Rürup, Bert (1997): Perspektiven der Pensionsversicherung in Österreich, Darmstadt Siegel, Jeremy J. (1998): Stocks for the Long Run, 2. Auflage, New York: McGraw-Hill Sinn, Hans Werner. (2000): Why a Funded Pension System is Useful and Why it is not Useful, in: International Tax and Public Finance, Jg. 7, Nr. 4-5, S. 389-4IO

Statistik Austria (Hg.) (2003): Statistisches Jahrbuch 2003, Wien

Statistik Austria (Hg.) (2004): Statistisches Jahrbuch 2004, Wien

Tichy, Gunther (1988): Ist die Altersvorsorge unfinanzierbar?, in: Die Presse vom I4.II.I988, S. 6

Tichy, Gunther (1999): Sechs Thesen zur Pensionspolitik für das 2I. Jahrhundert, in: Löffler, Heinz/Streissler, Erich (Hg.), Sozialpolitik und Ökologieprobleme der Zukunft. Festsymposium der Österreichischen Akademie der Wissenschaften anlässlich ihres I50jährigen Jubiläums, Wien, S. 22I-226

Tichy, Gunther (2003): Erfordert die Informationsgesellschaft flexiblere Arbeitsmärkte?, in: Perspektiven der Wirtschaftspolitik, Jg. 4, H. I, S. 29-4I

Tichy, Gunther (2004a): Der Wandel von der "Sozialdemokratischen Geborgenheit« zur »Neuen Unsicherheit« - Eine vernachlässigte Ursache der Stagnation, in: Wirtschaft und Gesellschaft, Jg. 30, H. I, S. 9-26

Tichy, Gunther (2004b): Der hochgespielte Generationenkonflikt - Ein Spiel mit dem Feuer, in: Zukunftsforum Österreich (Hg.), Generationen - Konflikt oder Harmonie? Sozialer Zusammenhalt zur Sicherung der Zukunft, Wien, S. 309-340

Tichy, Gunther (2005a): Armut in Österreich, in Acham, Karl (Hg.), Soziale und kulturelle Herausforderungen des 2I. Jahrhunderts, Wien, S. 55-63

Tichy, Gunther (2005b): Die »Neue Unsicherheit« als Ursache der europäischen Stagnation, in: Perspektiven der Wirtschaftspolitik, Jg. 6, H. 3, S. 385-407

Tichy, Gunther (2005c): Warum braucht Europa eine eigenständige Wirtschaftsverfassung?, in: Hödl, Erich (Hg.), Europäische Wirtschafts- und Gesellschaftsordnung, Marburg, S. 56-76

Westerheide, Peter (2000): Kosten der privaten Altersvorsorge. Private Rentenversicherungen und Fondspläne im Vergleich, Gütersloh

Williamson, John B. (200I): Future Prospects for Notional Defined Contribution Schemes, in: CESifo Forum, Jg. 2, Nr. 4, S. 19-24

Wissenschaftlicher Beirat beim Bundesministerium für Wirtschaft (1998): Grundlegende Reform der gesetzlichen Rentenversicherung, Bonn

Zöchling, Christa (2003): Romantische Gefühle, nüchterne Einsicht, in: profil Nr. 4I vom 06.10.2003, S. 28 